\title{
Addressing physician barriers to administering cyclin-dependent kinases 4 and 6 inhibitors in first-line treatment of hormone receptor- positive, human epidermal growth factor receptor 2-negative advanced breast cancer
}

This article was published in the following Dove Press journal: Cancer Management and Research

\section{Reshma L Mahtani Charles L Vogel}

Sylvester Comprehensive Cancer Center, University of Miami Health System, Deerfield Beach, FL, USA
Correspondence: Reshma L Mahtani Sylvester Comprehensive Cancer Center, University of Miami Health System, II 92 East Newport Center Drive, Deerfield Beach, FL 33442, USA

Tel +l 9546983639

Fax + I 954 57। 0118

Email rmahtani@miami.edu

\begin{abstract}
Combination therapy with a cyclin-dependent kinases 4 and 6 (CDK4/6) inhibitor and an aromatase inhibitor (AI) for first-line treatment of postmenopausal women with advanced breast cancer ( $\mathrm{ABC}$ ) has demonstrated improvement in progression-free survival (PFS) over AI monotherapy without adding substantial toxicity. However, CDK4/6 inhibitor plus AI therapy is not uniformly used as first-line therapy for $\mathrm{ABC}$, indicating that barriers to $\mathrm{CDK} 4 / 6$ inhibitor use exist. Such barriers may include the following perceptions: patients with bone-only metastases, with a long disease-free interval, or who are older may respond to AI monotherapy and may not benefit from a CDK4/6 inhibitor; tumor response rates may be lower and delayed with CDK4/6 inhibitor plus AI therapy than chemotherapy; the increased incidence of adverse events with CDK4/6 inhibitor plus AI therapy outweighs benefits; and the cost of CDK4/6 inhibitors may be prohibitive. Some of these barriers are addressed with data from follow-up analyses of CDK4/6 inhibitor trials, which have shown a PFS benefit of combination therapy in all subgroups assessed, including older patients, those with bone-only metastatic disease, and those with a long disease-free interval. Tumor response rates with CDK4/6 inhibitor plus AI therapy are comparable to those with first-line cytotoxic chemotherapy. Finally, adverse events associated with CDK4/6 inhibitor plus AI therapy are manageable and occur with decreasing severity during treatment, with similar reports of quality of life to those with AI monotherapy. These data support CDK4/6 inhibitor plus AI therapy as the standard of care in first-line treatment of ABC.

Keywords: aromatase inhibitor, ribociclib, palbociclib, abemaciclib
\end{abstract}

\section{Introduction}

Breast cancer is the most frequently diagnosed cancer and a leading cause of cancer deaths in women in the United States, with an estimated 266,120 new cases and 40,920 deaths in $2018 .{ }^{1}$ Approximately $71 \%$ of breast cancer cases are hormone receptorpositive $(\mathrm{HR}+$; estrogen receptor-positive $[\mathrm{ER}+]$ or progesterone receptor-positive) and human epidermal growth factor receptor 2-negative (HER2-). ${ }^{2}$ In HR+, HER2advanced postmenopausal breast cancer, aromatase inhibitor (AI) monotherapy is the standard-of-care treatment. ${ }^{3}$ However, progression is inevitable in the advanced setting, with most patients progressing on AI monotherapy within 13-16 months of treatment, as demonstrated in published studies. ${ }^{4,5}$ Thus, providing a first-line therapy that extends 
the duration of response to treatment while maintaining quality of life (QoL) is critical for this population of patients with advanced breast cancer (ABC).

Five randomized Phase II or III trials of the cyclindependent kinases 4 and 6 (CDK4/6) inhibitors palbociclib, ribociclib, and abemaciclib in combination with an AI have provided evidence of prolonged progression-free survival (PFS) with combination therapy compared with AI monotherapy. ${ }^{6-10}$ CDK4/6 inhibitors have been approved for use since palbociclib was approved in 2015 on the basis of PALOMA-1 trial results. ${ }^{11-13}$ However, many physicians continue to prescribe AI monotherapy or chemotherapy to patients with $\mathrm{HR}+, \mathrm{HER} 2-$ metastatic breast cancer in the first-line setting. ${ }^{14,15}$ Here, we briefly review the overall findings from clinical trials of CDK $4 / 6$ inhibitors in the first-line setting, present physician barriers to use of CDK4/6 inhibitor combination therapy in the first-line setting, and review current data that address these barriers.

\section{Addressing barriers to use of CDK4/6 inhibitor combination therapy in the first-line setting}

Five clinical trials, including four Phase III trials, have investigated the use of CDK4/6 inhibitors in combination with an AI in first-line treatment of $\mathrm{HR}+, \mathrm{HER} 2-\mathrm{ABC} .^{6-10,16}$ Although these trials enrolled similar patient populations overall, with some notable exceptions (the MONALEESA-7 trial [ribociclib] included only premenopausal women), there are certain patient characteristics that were represented to a greater extent in some trials than others. For example, there were more Asian patients in the MONARCH 3 trial (abemaciclib) and MONALEESA-7 trial (ribociclib) than other trials (Table 1)..$^{7,8,10,16}$ Thus, caution is advised when comparing individual trial efficacy and safety because conclusions from such comparisons are limited by differences in trial design and patient population.

The overall efficacy and safety of CDK4/6 inhibitors in combination with AIs in the first-line setting are summarized in Tables 2 and 3. As of the latest interim data cutoff dates for the Phase III trials, the median PFS in patients receiving a $\mathrm{CDK} 4 / 6$ inhibitor in combination with an $\mathrm{AI}$ ranged from 25.3 to 27.6 months (the median PFS was not reached at a median follow-up of 17.8 months in the MONARCH 3 trial). ${ }^{8-10,17}$ Median PFS for the AI control group ranged from 13.0 to 16.0 months. ${ }^{8-10,17}$ Combination therapy with CDK4/6 inhibitors was associated with an increased rate of asymptomatic neutropenia vs AI monotherapy, as well as other hematologic adverse events (AEs), nausea, diarrhea, fatigue, and alopecia (Table 3$).^{7-10}$

Table I Baseline characteristics of patients enrolled in trials of CDK4/6 inhibitors plus an Al for first-line treatment of HR+, HER2$A B C$

\begin{tabular}{|c|c|c|c|c|c|c|c|c|}
\hline & \multicolumn{2}{|c|}{ MONALEESA-2 ${ }^{16}$} & \multicolumn{2}{|c|}{ MONALEESA-7 ${ }^{10}$} & \multicolumn{2}{|c|}{ MONARCH $3^{8}$} & \multicolumn{2}{|c|}{ PALOMA-2 ${ }^{7}$} \\
\hline & $\begin{array}{l}\text { RIB + LET } \\
(n=334)\end{array}$ & $\begin{array}{l}\text { PBO + LET } \\
(n=334)\end{array}$ & $\begin{array}{l}\text { RIB + TAM } \\
\text { or NSAI } \\
(n=335)\end{array}$ & $\begin{array}{l}\text { PBO + TAM } \\
\text { or NSAI } \\
(n=337)\end{array}$ & $\begin{array}{l}\text { ABE + } \\
\text { NSAI } \\
(n=328)\end{array}$ & $\begin{array}{l}\text { PBO + } \\
\text { NSAI } \\
(n=165)\end{array}$ & $\begin{array}{l}\text { PAL } \\
+ \text { LET } \\
(n=444)\end{array}$ & $\begin{array}{l}\text { PBO } \\
+ \text { LET } \\
(n=222)\end{array}$ \\
\hline Median age, years & 62 & 63 & 43 & 45 & 63 & 63 & 62 & 61 \\
\hline ECOG PS, \% & & & & & & & & \\
\hline 0 & 61 & 60 & 73 & 76 & 58 & 63 & 58 & 46 \\
\hline 1 & 39 & 40 & 26 & 23 & 42 & 37 & 40 & 53 \\
\hline 2 & 0 & 0 & 0 & $<1$ & 0 & 0 & 2 & 1 \\
\hline \multicolumn{9}{|l|}{ Race, \% } \\
\hline White & 81 & 84 & 56 & 60 & 57 & 62 & 77 & 78 \\
\hline Asian & 8 & 7 & 30 & 29 & 31 & 27 & 15 & 14 \\
\hline Other or unknown & 11 & 9 & 15 & II & 3 & 4 & 8 & 9 \\
\hline Visceral disease, \% & 59 & 59 & 58 & 56 & 52 & 54 & 48 & 50 \\
\hline Bone-only disease, \% & 21 & 23 & 24 & 23 & 21 & 24 & 23 & 22 \\
\hline $\begin{array}{l}\text { De novo metastatic } \\
\text { disease, \% }\end{array}$ & 34 & 34 & 41 & 40 & 41 & 37 & 38 & 36 \\
\hline $\begin{array}{l}\text { Prior (neo)adjuvant } \\
\text { ET, \% }\end{array}$ & 52 & 51 & 38 & 42 & 46 & 48 & 56 & 57 \\
\hline Prior TAM, \% & 42 & 43 & Not reported & Not reported & Not reported & Not reported & $47^{a}$ & $44^{a}$ \\
\hline Prior CT, \% & $44^{\mathrm{b}}$ & $43^{b}$ & $4 I^{\mathrm{b}}$ & $4 I^{\mathrm{b}}$ & 38 & 40 & $48^{\mathrm{b}}$ & $49^{b}$ \\
\hline
\end{tabular}

Notes: an the adjuvant setting only. 'In the neoadjuvant or adjuvant setting.

Abbreviations: ABC, advanced breast cancer; ABE, abemaciclib; Al, aromatase inhibitor; CDK4/6, cyclin-dependent kinases 4 and 6; CT, chemotherapy; ECOG PS, Eastern Cooperative Oncology Group performance status; ET, endocrine therapy; HER2-, human epidermal growth factor receptor 2-negative; HR+, hormone receptor-positive; LET, letrozole; NR, not reported; NSAI, nonsteroidal aromatase inhibitor; PAL, palbociclib; PBO, placebo; RIB, ribociclib; TAM, tamoxifen. 
Table 2 Overall efficacy reported in trials of CDK4/6 inhibitors plus an Al for first-line treatment of HR+, HER2- ABC

\begin{tabular}{|c|c|c|c|c|c|c|c|c|}
\hline & \multicolumn{2}{|c|}{ MONALEESA-2 ${ }^{9}$} & \multicolumn{2}{|c|}{ MONALEESA-7 ${ }^{10}$} & \multicolumn{2}{|c|}{ MONARCH $3^{8}$} & \multicolumn{2}{|l|}{ PALOMA-2 $^{7}$} \\
\hline & $\begin{array}{l}\text { RIB + } \\
\text { LET } \\
(n=334)\end{array}$ & $\begin{array}{l}\text { PBO } \\
+ \text { LET } \\
(n=334)\end{array}$ & $\begin{array}{l}\text { RIB + TAM } \\
\text { or NSAI } \\
(n=335)\end{array}$ & $\begin{array}{l}\text { PBO + TAM } \\
\text { or NSAI } \\
(n=337)\end{array}$ & $\begin{array}{l}\text { ABE + } \\
\text { NSAI } \\
(n=328)\end{array}$ & $\begin{array}{l}\text { PBO + } \\
\text { NSAI } \\
(n=165)\end{array}$ & $\begin{array}{l}\text { PAL + LET } \\
(n=444)\end{array}$ & $\begin{array}{l}\text { PBO } \\
+ \text { LET } \\
(n=222)\end{array}$ \\
\hline Median PFS, months & 25.3 & 16.0 & 23.8 & 13.0 & Not reached & 14.7 & 24.8 & 14.5 \\
\hline Hazard ratio $(95 \% \mathrm{Cl})$ & \multicolumn{2}{|c|}{$0.57(0.46-0.70)$} & \multicolumn{2}{|c|}{$0.55(0.44-0.69)$} & \multicolumn{2}{|c|}{$0.54(0.4 I-0.72)$} & \multicolumn{2}{|c|}{$0.58(0.46-0.72)$} \\
\hline$P$-value & \multicolumn{2}{|l|}{$9.63 \times 10^{-8}$} & \multicolumn{2}{|l|}{$<0.000$ I } & \multicolumn{2}{|l|}{$2.1 \times 10^{-5}$} & \multicolumn{2}{|l|}{$<0.001$} \\
\hline CBR, \% & $79.9^{a}$ & $73.1^{a}$ & 79.1 & 69.7 & $78.0^{\mathrm{b}}$ & $71.5^{\mathrm{b}}$ & $84.9^{c}$ & $70.3^{c}$ \\
\hline ORR, \% ${ }^{d}$ & 42.5 & 28.7 & 40.9 & 29.7 & 48.2 & 34.5 & 42.1 & 34.7 \\
\hline
\end{tabular}

Notes: ${ }^{\mathrm{a} C B R}=\mathrm{CR}+\mathrm{PR}+\mathrm{SD}$ for $\geq 24$ weeks $+\mathrm{NCRNPD}$ for $\geq 24$ weeks. ${ }^{\mathrm{b}} \mathrm{CBR}=\mathrm{CR}+\mathrm{PR}+\mathrm{SD}$ for $\geq 6$ months. ${ }^{\mathrm{c} C B R}=\mathrm{CR}+\mathrm{PR}+\mathrm{SD}$ for $\geq 24$ weeks. ${ }^{\mathrm{d} O R R}=\mathrm{CR}+\mathrm{PR}$. Abbreviations: $A B C$, advanced breast cancer; $A B E$, abemaciclib; $A l$, aromatase inhibitor; $C B R$, clinical benefit rate; $C D K 4 / 6$, cyclin-dependent kinases 4 and 6; $C R$, complete response; HER2-, human epidermal growth factor receptor 2-negative; HR+, hormone receptor-positive; LET, letrozole; NCRNPD, neither complete response nor progressive disease; NSAI, nonsteroidal aromatase inhibitor; ORR, objective response rate; PAL, palbociclib; PBO, placebo; PFS, progression-free survival; PR, partial response; RIB, ribociclib; SD, stable disease; TAM, tamoxifen.

Table 3 Overall safety reported in trials of CDK4/6 inhibitors plus an Al for first-line treatment of HR+, HER2- ABC

\begin{tabular}{|c|c|c|c|c|c|c|c|c|}
\hline \multirow{2}{*}{$\begin{array}{l}\text { Adverse events, } \\
\text { any grade, in } \geq 30 \% \\
\text { of patients in any } \\
\text { treatment group, } \%\end{array}$} & \multicolumn{2}{|c|}{ MONALEESA- $2^{9}$} & \multicolumn{2}{|c|}{ MONALEESA-7 ${ }^{10}$} & \multicolumn{2}{|l|}{ MONARCH $3^{8}$} & \multicolumn{2}{|l|}{ PALOMA-2 ${ }^{7}$} \\
\hline & $\begin{array}{l}\text { RIB + } \\
\text { LET } \\
(n=334)\end{array}$ & $\begin{array}{l}\text { PBO + } \\
\text { LET } \\
(n=330)\end{array}$ & $\begin{array}{l}\text { RIB + TAM } \\
\text { or NSAI } \\
(n=335)\end{array}$ & $\begin{array}{l}\text { PBO + TAM } \\
\text { or NSAI } \\
(n=337)\end{array}$ & $\begin{array}{l}\text { ABE + NSAI } \\
(n=327)\end{array}$ & $\begin{array}{l}\text { PBO + NSAI } \\
(n=161)\end{array}$ & $\begin{array}{l}\text { PAL + LET } \\
(n=444)\end{array}$ & $\begin{array}{l}\text { PBO + } \\
\text { LET } \\
(n=222)\end{array}$ \\
\hline Neutropenia $^{a}$ & 77 & 6 & 76 & 8 & 41 & 2 & 80 & 6 \\
\hline Nausea & 53 & 31 & 32 & 20 & 39 & 20 & 35 & 26 \\
\hline Infections (pooled) & $50^{16}$ & $42^{16}$ & Not reported & Not reported & 39 & 29 & $60^{12}$ & $42^{12}$ \\
\hline Fatigue & 41 & 32 & 24 & 25 & 40 & 32 & 37 & 27 \\
\hline Diarrhea & 38 & 25 & 20 & 19 & 81 & 30 & 26 & 19 \\
\hline Vomiting & 34 & 17 & 19 & 17 & 28 & 12 & 16 & 17 \\
\hline Alopecia & 34 & 16 & 19 & 12 & 27 & 11 & 33 & 16 \\
\hline Leukopenia $^{\mathrm{b}}$ & 33 & 5 & 31 & 6 & 21 & 2 & 39 & 2 \\
\hline Arthralgia & 33 & 33 & 30 & 27 & Not reported & Not reported & 33 & 34 \\
\hline Hot flush ${ }^{c}$ & 25 & 25 & 34 & 34 & Not reported & Not reported & 21 & 31 \\
\hline Anemia $^{d}$ & 21 & 6 & 21 & 10 & 28 & 5 & 24 & 9 \\
\hline
\end{tabular}

Notes: aln the MONALEESA-2 trial, neutropenia also included granulocytopenia and decreased neutrophil count. In the PALOMA-2 trial, neutropenia also included decreased neutrophil count. 'In the PALOMA-2 and MONALEESA-2 trials, leukopenia also included decreased white blood cell count. In the MONALEESA-7 and MONALEESA-2 trials, hot flash was reported. In the MONALEESA-2 trial, anemia also included decreased hemoglobin and macrocytic anemia. Superscripted numbers represent reference citations.

Abbreviations: ABC, advanced breast cancer; ABE, abemaciclib; Al, aromatase inhibitor; CDK4/6, cyclin-dependent kinases 4 and 6; HER2-, human epidermal growth factor receptor 2-negative; HR+, hormone receptor-positive; LET, letrozole; NSAI, nonsteroidal aromatase inhibitor; PAL, palbociclib; PBO, placebo; RIB, ribociclib; TAM, tamoxifen.

Abemaciclib therapy was associated with a lower incidence of neutropenia (41\%) compared to ribociclib (77\%) and palbociclib $(80 \%)$ therapy, but also a higher incidence of diarrhea (81\%) compared to palbociclib (26\%) and ribociclib (38\%) therapy. ${ }^{7-9}$ Ribociclib therapy was associated with a higher incidence of nausea (53\%) compared to palbociclib (35\%) and abemaciclib (39\%) therapy. ${ }^{7-9}$ Any decision regarding cancer therapy should be made considering the potential risks and benefits of a given regimen with respect to the individual patient. Below, we address important points physicians should consider regarding use of CDK4/6 inhibitor plus AI therapy.

\section{Prolonged response to Al monotherapy}

Physician barrier: certain patients may respond well to Al monotherapy and may not benefit enough from the addition of a CDK4/6 inhibitor to outweigh safety concerns

It is important to consider individual patient characteristics when treating $\mathrm{ABC}$. In older patients, those who have boneonly metastatic disease or those who have a long disease-free interval after completion of adjuvant therapy, AI monotherapy 
may be highly efficacious, with median PFS responses greater than the previously mentioned 13.8-16.0 months demonstrated in first-line AI monotherapy studies. However, results from randomized trials indicate that CDK4/6 inhibitor combination therapy provides greater benefit than AI monotherapy in each of these subgroups, with similar safety profiles in older and younger patients (Table 4).

\section{Older patients}

In a pooled analysis of the PALOMA-1 and PALOMA-2 trials, the median PFS in patients in the placebo plus letrozole group was longer in patients aged 65-74 years $(21.8$ months; $n=94)$ than that in patients aged $<65$ years $(12.3$ months; $n=183$ ); although, in patients aged $\geq 75$ years in the placebo plus letrozole group $(n=26)$, the median PFS was only 10.9 months. ${ }^{18}$ Nevertheless, palbociclib plus letrozole therapy resulted in a $69 \%$ (median PFS, not reached) and $34 \%$ (median PFS, 27.5 months) reduction in risk of disease progression in patients aged $\geq 75$ years or aged $65-74$ years, respectively, compared to letrozole monotherapy. Hematologic AEs, infections, and decreased appetite were more commonly observed in patients $\geq 65$ years old than $<65$ years old. ${ }^{18}$

A subgroup analysis in patients aged $\geq 65$ years in the MONARCH 3 trial, after a median follow-up of 17.8 months, reported a $43 \%$ reduction in risk of progression with abemaciclib plus nonsteroidal AI (NSAI) therapy compared to placebo plus NSAI therapy, consistent with findings in the overall population. ${ }^{8}$

In a subgroup analysis in patients aged $\geq 65$ years in the MONALEESA-2 trial, after a median follow-up of 15.3 months, the median PFS in older patients in the placebo plus letrozole group (18.4 months; $n=145$ ) was longer than that in younger (aged <65 years) patients (13.0 months; $n=189) .{ }^{16,19}$ However, older patients exhibited a 39\% reduction in risk of progression with ribociclib plus letrozole therapy (median PFS, not reached) compared to placebo plus letrozole therapy (median PFS, 18.4 months), consistent with findings in the overall population. ${ }^{19}$ Rates of AEs, including neutropenia, were similar in older and younger patients. ${ }^{19}$

Finally, a pooled retrospective subgroup analysis of the registration studies that led to approval of the three CDK4/6 inhibitors in older and younger patients conducted by the US Food and Drug Administration confirmed a PFS benefit of CDK4/6 inhibitor plus AI therapy in older patients across trial populations. ${ }^{20}$ Therefore, there are no data to support withholding CDK4/6 inhibitor plus AI therapy based solely on age.

\section{Patients with bone-only disease}

The overall prevalence of bone-only metastases in patients with $\mathrm{ABC}$ in the first-line setting ranges from $\sim 15 \%$ to $35 \%$, according to a retrospective review of medical records and

Table 4 Efficacy reported in subgroups from trials of CDK4/6 inhibitors plus an Al for first-line treatment of HR+, HER2- ABC

\begin{tabular}{|c|c|c|c|c|c|c|}
\hline & \multicolumn{2}{|c|}{ MONALEESA-2 ${ }^{16,19,26,73}$} & \multicolumn{2}{|l|}{ MONARCH $3^{8}$} & \multicolumn{2}{|c|}{ PALOMA-2 ${ }^{18,27,46}$} \\
\hline & RIB + LET & PBO + LET & ABE + NSAI & PBO + NSAI & PAL + LET & PBO + LET \\
\hline Older patients, cutoff $(n)$ & $\geq 65$ years $(150)$ & $\geq 65$ years $(145)$ & $\begin{array}{l}\geq 65 \text { years (not } \\
\text { reported) }\end{array}$ & $\begin{array}{l}\geq 65 \text { years (not } \\
\text { reported) }\end{array}$ & $\begin{array}{l}\geq 65 \text { and }<75 \\
\text { years }(162)^{\mathrm{a}}\end{array}$ & $\begin{array}{l}\geq 65 \text { and }<75 \\
\text { years }(94)^{\mathrm{a}}\end{array}$ \\
\hline Median follow-up, months & \multicolumn{2}{|l|}{15.3} & \multicolumn{2}{|l|}{17.8} & \multicolumn{2}{|l|}{ Not reported } \\
\hline Median PFS, months & Not reached & 18.4 & Not reported & Not reported & $27.5^{\mathrm{a}}$ & $21.8^{\mathrm{a}}$ \\
\hline Hazard ratio $(95 \% \mathrm{Cl})$ & \multicolumn{2}{|c|}{$0.608(0.394-0.937)$} & \multicolumn{2}{|l|}{$0.57(0.36-0.90)$} & \multicolumn{2}{|c|}{$0.66^{\mathrm{a}}(0.45-0.97)$} \\
\hline$P$-value & \multicolumn{2}{|l|}{ Not reported } & \multicolumn{2}{|l|}{ Not reported } & \multicolumn{2}{|l|}{$<0.016^{\mathrm{a}}$} \\
\hline $\begin{array}{l}\text { Patients with bone-only disease, } \\
\mathrm{n}\end{array}$ & 69 & 78 & 70 & 39 & 103 & 48 \\
\hline Median follow-up, months & \multicolumn{2}{|l|}{15.3} & \multicolumn{2}{|l|}{17.8} & \multicolumn{2}{|l|}{23} \\
\hline Median PFS, months & Not reached & 15.3 & Not reached & Not reached & Not reached & 11.2 \\
\hline Hazard ratio $(95 \% \mathrm{Cl})$ & \multicolumn{2}{|c|}{$0.690(0.38|-| .249)$} & \multicolumn{2}{|l|}{$0.58(0.27-1.25)$} & \multicolumn{2}{|c|}{$0.36(0.22-0.59)$} \\
\hline$P$-value & \multicolumn{2}{|l|}{ Not reported } & \multicolumn{2}{|l|}{ Not reported } & \multicolumn{2}{|l|}{$<0.0001$} \\
\hline $\begin{array}{l}\text { Patients with long disease-free } \\
\text { interval, cutoff }(n)\end{array}$ & $>48$ months (54) & $\begin{array}{l}>48 \text { months } \\
\text { (49) }\end{array}$ & $\begin{array}{l}\geq 36 \text { months } \\
\text { (94) }\end{array}$ & $\begin{array}{l}\geq 36 \text { months } \\
(40)\end{array}$ & $\begin{array}{l}>12 \text { months } \\
(178)\end{array}$ & $\begin{array}{l}>12 \text { months } \\
(93)\end{array}$ \\
\hline Median follow-up, months & \multicolumn{2}{|l|}{26.4} & \multicolumn{2}{|l|}{17.8} & \multicolumn{2}{|l|}{23} \\
\hline Median PFS, months & 29.6 & 19.2 & Not reached & Not reached & 25.4 & 13.8 \\
\hline Hazard ratio $(95 \% \mathrm{Cl})$ & \multicolumn{2}{|c|}{$0.496(0.274-0.898)$} & \multicolumn{2}{|l|}{$0.83(0.46-1.52)$} & \multicolumn{2}{|c|}{$0.52(0.37-0.73)$} \\
\hline$P$-value & \multicolumn{2}{|l|}{ Not reported } & \multicolumn{2}{|l|}{ Not reported } & \multicolumn{2}{|l|}{$<0.0001$} \\
\hline
\end{tabular}

Notes: aResults in older patients reported from a pooled analysis of PALOMA-I and PALOMA-2 trial participants.

Abbreviations: $A B C$, advanced breast cancer; ABE, abemaciclib; Al, aromatase inhibitor; CDK4/6, cyclin-dependent kinases 4 and 6; HER2-, human epidermal growth factor receptor 2-negative; HR+, hormone receptor-positive; LET, letrozole; NSAI, nonsteroidal AI; PAL, palbociclib; PBO, placebo; PFS, progression-free survival; RIB, ribociclib. 
a prospective tumor registry study. ${ }^{21,22}$ In Phase III trials of CDK4/6 inhibitors in combination with fulvestrant in the second-line setting, $24 \%-27 \%$ of patients had bone-only metastases. $^{23,24}$ Patients enrolled in the MONARCH 2 trial with bone-only metastases experienced a benefit from addition of abemaciclib after a median follow-up of 19.5 months (median PFS, 24.0 months [abemaciclib] vs 16.6 months [placebo]; hazard ratio, 0.544 [95\% CI, 0.355-0.834]). ${ }^{23,25}$ Similarly, in the PALOMA-3 trial, patients with bone-only metastases demonstrated a PFS benefit with the addition of palbociclib (median PFS, 14.3 months [palbociclib plus fulvestrant] vs 9.2 months [placebo plus fulvestrant]; $P<0.05) .{ }^{24}$ In Phase III clinical trials of CDK4/6 inhibitors in combination with AIs in the first-line setting, a similar proportion of participants had enrolled with bone-only metastatic disease (range, 21\%-24\%). ${ }^{7,8,10,16}$ These trials reported hazard ratios favoring combination therapy in patients with bone-only disease, reporting between $31 \%$ and $64 \%$ reduction in risk of disease progression (Table 4). ${ }^{8,26,27}$

Although all pivotal trials lack long-term follow-up, preliminary data for patients with bone-only disease are promising and consistent in favoring CDK4/6 inhibitor plus AI therapy over AI monotherapy. The median PFS was not reached in the CDK4/6 inhibitor plus AI treatment groups of the PALOMA-2, MONARCH 3, and MONALEESA-2 trials at the current data cutoffs for these analyses (median follow-up lengths of 23 months, 17.8 months, and 15.3 months, respectively), restricting any numerical comparisons of PFS between treatment groups. ${ }^{8,16,26,27}$ However, the lower limit of the $95 \%$ CI of median PFS in patients on palbociclib plus letrozole combination therapy in the PALOMA-2 trial suggests that the true median PFS will likely be $>24.8$ months in these patients. Comparatively, the median PFS in the letrozole plus placebo group of the PALOMA-2 trial was 11.2 months (95\% CI, 8.2-22.0 months). ${ }^{27}$ Results from the MONALEESA-2 (ribociclib) and MONARCH 3 (abemaciclib) trials are still immature. ${ }^{8,26}$ Given the consistent PFS benefit of CDK4/6 inhibitor plus AI therapy demonstrated in patients with bone-only disease, it is reasonable to consider combination therapy for these patients.

\section{Patients with a long disease-free interval after neoadjuvant or adjuvant therapy}

Patients with a long disease-free interval after completion of adjuvant therapy can experience a longer median PFS with
AI monotherapy in first-line ABC compared to patients with a short disease-free interval. ${ }^{27,28}$ In the PALOMA-2, MONALEESA-2, and MONARCH 3 trials, disease-free interval was calculated as the time from the end of the last neoadjuvant or adjuvant therapy received to randomization. . $^{87,28}$ In patients receiving AI monotherapy, the median PFS was greater in patients with a long disease-free interval vs those with a short disease-free interval in the MONALEESA-2 trial ( $>48$ months vs $\leq 48$ months; 8.3-month difference) and PALOMA- 2 trial ( $>12$ months vs $\leq 12$ months; 2 .8-month difference). ${ }^{27,28}$ However, even in these patients, a PFS benefit of CDK4/6 inhibitor plus AI therapy was observed.

In the MONALEESA-2 trial, after a median follow-up of 26.4 months, an exploratory subgroup analysis of 298 patients with prior neoadjuvant or adjuvant endocrine therapy (ET) demonstrated benefit of ribociclib plus letrozole combination therapy over letrozole monotherapy in patients with a treatment-free interval of $>24,>36$, or $>48$ months $(54.5 \%, 49.3 \%$, and $50.4 \%$ reduction in risk of disease progression or death with ribociclib, respectively). ${ }^{28}$

In the PALOMA-2 trial, results from a subgroup analysis of patients with a disease-free interval of $>12$ months at a median follow-up of 23 months reported the median PFS in the palbociclib plus letrozole group as 25.4 months (95\% CI, 22.2 months - not reached; $n=178$ ) vs 13.8 months (95\% CI, 9.6-18.2 months; $n=93$ ) in the placebo plus letrozole group, a $48 \%$ reduction in risk of progression. ${ }^{27}$ A similar analysis of the MONALEESA-7 trial at a median follow-up of 19.2 months demonstrated a $25 \%$ reduction in risk of progression with ribociclib plus $\mathrm{NSAI} /$ tamoxifen combination therapy compared to NSAI/ tamoxifen in 25 premenopausal women with a disease-free interval of $>12$ months. ${ }^{10}$

In contrast, the MONARCH 3 trial reported an exploratory subgroup analysis of 134 patients with a disease-free interval of $\geq 36$ months at a median follow-up of 17.8 months. ${ }^{8}$ This analysis did not demonstrate a difference between groups, with the median PFS not reached in either group, thus highlighting the need for longer follow-up to demonstrate a PFS benefit of abemaciclib plus AI combination therapy in this group of patients. ${ }^{8}$

All these analyses report results at interim data cutoffs, often with limited sample sizes. Final analyses with longer follow-up may more clearly define differences in efficacy between CDK4/6 inhibitor plus AI therapy and AI monotherapy in these patients. 


\section{Tumor response rates with chemotherapy and CDK4/6 inhibitor combination therapy}

Physician barrier: tumor response rates with chemotherapy may be higher than with CDK4/6 inhibitors in the first-line setting

National guidelines recommend use of ET over chemotherapy in the first-line setting unless the disease is immediately lifethreatening or patients have rapid visceral recurrence during adjuvant ET. ${ }^{3}$ Tumor response rates were reported in Phase III trials of CDK4/6 inhibitor plus AI therapy; however, except in the MONALEESA-7 trial, these trials excluded patients who progressed within 12 months after adjuvant NSAI therapy. ${ }^{7,810,16}$ Patients with immediately life-threatening disease were also excluded in the MONALEESA-7, MONARCH 3 , and PALOMA-2 trials, and patients with inflammatory breast cancer were excluded in the MONALEESA-2, MONALEESA-7, and MONARCH 3 trials. ${ }^{7,8,10,16,29}$ Interpretation of tumor response rates should take into consideration these trial characteristics.

In postmenopausal women with $\mathrm{HER} 2-\mathrm{ABC}$ in the firstline setting, overall response rates (ORRs) for single-agent docetaxel or $n a b$-paclitaxel ranged from $35 \%$ to $49 \%$, while ORRs in Phase III trials of CDK4/6 inhibitors ranged from $29 \%$ to $35 \%$ for AI monotherapy and from $42 \%$ to $48 \%$ for CDK 4/6 inhibitor plus AI therapy., ${ }^{4,-9,30,31}$ In these same trials, CBRs ranged from $58 \%$ to $80 \%$ for single-agent docetaxel or $n a b$-paclitaxel, $70 \%$ to $73 \%$ for AI monotherapy, and $78 \%$ to $85 \%$ for CDK4/6 inhibitor plus AI therapy. ${ }^{4,79,30,31}$

Tumor responses with single-agent docetaxel or paclitaxel have been reported to occur within the first 6-12 weeks of treatment in patients with $\mathrm{ABC} .{ }^{32,33}$ Tumor response to CDK4/6 inhibitor-based therapy also tends to occur within the first few months of treatment. In the MONALEESA-2 trial, an improvement in tumor response with ribociclib plus letrozole combination therapy compared to AI monotherapy was observed at 8 weeks. ${ }^{28}$ In the PALOMA-3 trial, which assessed palbociclib plus fulvestrant as second-line therapy, the median time to response was 16 weeks. ${ }^{34}$

Patients with visceral metastases were enrolled in all four Phase III clinical trials of first-line CDK4/6 inhibitor plus AI therapy (PALOMA-2: 73\%; MONALEESA-2: 59\%; MONARCH 3: 53\%; MONALEESA-7: 57\%) and in the FALCON trial, which assessed first-line fulvestrant monotherapy vs anastrozole monotherapy in ET-naive postmenopausal patients with $\mathrm{HR}+\mathrm{ABC}$. $7,8,10,16,35$ Subgroup analyses have shown a PFS benefit with CDK4/6 inhibitor plus AI therapy over AI monotherapy in patients with visceral metastases. In the PALOMA-2 trial, after a median follow-up of 23 months, palbociclib plus letrozole combination therapy demonstrated a 6.4-month greater median PFS in patients with visceral disease compared to letrozole monotherapy. ${ }^{27}$ Data from the MONALEESA-2 and MONARCH 3 trials are either immature or not inclusive of all visceral metastases, but both support similar trends as those observed in the PALOMA-2 trial. ${ }^{8,25,26}$

Among ET-naive patients in the FALCON trial, those with visceral metastases had similar PFS benefit with fulvestrant or anastrozole monotherapy. Among patients without visceral metastases in the FALCON trial, those administered fulvestrant had a longer median PFS compared to those administered anastrozole (fulvestrant: 22.3 months; anastrozole: 13.8 months). ${ }^{35}$ All of these data to date support the use of hormonal therapy as first-line therapy in patients with visceral metastases that are not immediately life-threatening.

\section{Tolerability and QoL with CDK4/6 inhibitors \\ Physician barrier: CDK4/6 inhibitor combination therapy is associated with increased AEs that may limit tolerability, negatively affect QoL, or outweigh efficacy benefits compared with endocrine monotherapy}

Neutropenia is the most common Grade 3/4 AE observed with palbociclib and ribociclib in combination with letrozole. Neutropenia is also observed with abemaciclib in combination with letrozole, although to a lesser extent (Table 3). Neutropenia associated with CDK4/6 inhibitor combination therapy is rarely associated with fever and is thought to be mechanistically different from neutropenia associated with chemotherapy. ${ }^{36} \mathrm{CDK} 4 / 6$ inhibitors induce cell-cycle arrest rather than apoptotic cell death in hematopoietic stem cells. ${ }^{36}$ The maintenance of these hematopoietic progenitor cell populations under CDK4/6 inhibition is hypothesized to allow for the rapid resolution of neutropenia associated with CDK4/6 inhibitors in clinical trials. ${ }^{36}$ Use of growth factors is not recommended with CDK4/6 inhibitor-induced neutropenia, except in the case of concurrent fever. ${ }^{37}$ Dose reductions and interruptions due to neutropenia occurred in the PALOMA-2 trial $(24.3 \%$ and $54.7 \%$ of patients, respectively) and MONALEESA-2 trial (31.1\% and 49.7\% of patients, respectively). ${ }^{38,39}$ However, discontinuation due to neutropenia was low in both the MONALEESA-2 
and PALOMA-2 trials $(0.9 \%$ and $1.1 \%$ of patients, respectively), indicating that neutropenia was manageable. ${ }^{38,39}$ An analysis of patients who underwent dose modification in the palbociclib group of the PALOMA-2 trial suggests that dose modification does not negatively affect PFS. ${ }^{38} \mathrm{CDK} 4 / 6$ inhibitor-induced neutropenia typically occurs early during treatment and is reversible..$^{8,39,40}$ Rates of Grade 3/4 neutropenia in the MONALEESA-2 trial decreased from $60.2 \%$ in the ribociclib plus letrozole group within the first 12 months of treatment to $16.8 \%$ after 18 months of treatment. ${ }^{39}$ In the MONARCH 3 trial, Grade 3/4 neutropenia was less commonly observed after the first two cycles of therapy, with incidences of $<5 \%$ in any given cycle. ${ }^{8}$

Among nonhematologic toxicities, diarrhea is the most common with abemaciclib combination therapy and is observed to a lesser extent with ribociclib or palbociclib combination therapy (Table 3). Diarrhea observed with CDK4/6 inhibitors is predominantly Grade 1 or $2 .^{7-10} \mathrm{~A}$ high rate of Grade 3 diarrhea is observed with abemaciclib therapy, but this can be managed with antidiarrheal medications. ${ }^{8,41}$

In addition to diarrhea, $\mathrm{CDK} 4 / 6$ inhibitor combination therapy is associated with nausea and vomiting, fatigue, infections, and alopecia (Table 3). These AEs are predominantly Grade 1 or $2 .^{7-10}$ Fatigue was reported in $24 \%-40 \%$ of patients with CDK4/6 inhibitor plus AI therapy vs $25 \%-32 \%$ with AI monotherapy. Alopecia is observed in $19 \%-34 \%$ of patients receiving CDK4/6 inhibitor plus AI therapy vs $11 \%-16 \%$ of patients receiving AI monotherapy. ${ }^{7-10}$ Grade 1 alopecia is defined as $<50 \%$ of the patient's hair falling out, with effects not noticeable from a distance. ${ }^{42} \mathrm{~A}$ small proportion of patients $(<3 \%)$ receiving palbociclib plus letrozole in the PALOMA-2 trial or receiving abemaciclib plus NSAI in the MONARCH 3 trial reported experiencing Grade 2 alopecia, where $\geq 50 \%$ of the patient's hair has fallen out, and a wig or other head covering is necessary to camouflage the hair loss. ${ }^{7,8,42}$ The MONALEESA-2 trial had a similar proportion of patients who experienced any grade of alopecia as the PALOMA-2 and MONARCH 3 trials. ${ }^{9}$

All approved CDK4/6 inhibitors are associated with laboratory abnormalities. ${ }^{8,11,12}$ In addition to being associated with decreased cell counts related to hematologic AEs, all CDK4/6 inhibitors are associated with increases in alanine aminotransferase (range, 33\%-48\%) and aspartate aminotransferase (range, 37\%-52\%), although liver function tests are currently only recommended with ribociclib and abemaciclib. ${ }^{8,11,12,41}$ Increases in liver aminotransferases are manageable with dose modification. ${ }^{8,16}$ Abemaciclib in combination with an NSAI is associated with increased creatinine compared to NSAI monotherapy, although this is not associated with impaired renal function. ${ }^{8}$

Prolongation of the QT interval has been reported with palbociclib and ribociclib in the PALOMA-2, MONALEESA-2, and MONALEESA-7 trial populations, and biweekly electrocardiogram monitoring is recommended through the beginning of the second month of ribociclib use. ${ }^{11}$ Because the QT interval can be altered by resting heart rate, the electrocardiogram reading must be corrected by heart rate for accurate interpretation. ${ }^{43}$ The QT interval can vary from beat to beat, so multiple readings should be performed to accurately assess the presence of QT interval prolongation. ${ }^{44}$ Fridericia's formula was used to correct the QT interval reading in the MONALEESA-2 and MONALEESA-7 trials and is recommended to be used when assessing the QT interval in patients who are initiating ribociclib therapy. ${ }^{11,16}$ In both the MONALEESA-2 and PALOMA-2 trials, the reported rate of QT interval prolongation was low, with only one patient in either trial experiencing a corrected QT interval (Fridericia's formula) of $\geq 500 \mathrm{~ms}^{39,45}$ In the MONALEESA-7 trial, five patients $(1 \%)$ in the ribociclib combination therapy group experienced a corrected QT interval (Fridericia's formula) of $>500$ milliseconds. ${ }^{10}$ Data on the effect of abemaciclib on QT interval prolongation have not yet been reported in the MONARCH 3 trial population. ${ }^{8}$

Patient reports of QoL in the PALOMA and MONALEESA trials suggest that QoL is not negatively affected by the above toxicities. In the PALOMA-2 trial, there was no difference in global health status, as measured by the EuroQol 5-domain questionnaire and visual analog scale. ${ }^{46}$ In the PALOMA-1/TRIO-18 trial, an analysis of pain from the Brief Pain Inventory tool found that pain severity and pain interference scores remained stable with a slight improvement in pain in both treatment groups and no overall differences between treatment groups. ${ }^{47}$

In the MONALEESA-2 and MONALEESA-7 trials, patient-reported outcomes were measured using the European Organisation for Research and Treatment of Cancer Quality of Life Questionnaire C30. ${ }^{10,48}$ In the MONALEESA-2 trial, no differences between groups were seen in global healthrelated QoL or symptom scales, and patients in the ribociclib group exhibited similar or greater reduction in pain than patients in the placebo group. ${ }^{48}$ In the MONALEESA-7 trial in premenopausal women, time to definitive deterioration of $\geq 10 \%$ in global health status/QoL score was improved with ribociclib plus ovarian suppression in combination with either an NSAI or tamoxifen compared to ovarian suppression with 
an NSAI or tamoxifen without ribociclib, and improvement in pain score from baseline was observed in the ribociclib group at 8 weeks. ${ }^{10}$ As of the development of this review, abemaciclib QoL data are not currently publicly available.

\section{Costs associated with CDK4/6 inhibitors \\ Physician barrier: prescribing CDK4/6 inhibitors with Al therapy may add prohibitive costs to treatment}

The starting dose of all three CDK4/6 inhibitors are priced at $>\$ 11,000$ per monthly dose without considering discounts or insurance. ${ }^{49-51}$ A cost-effectiveness analysis of palbociclib therapy in the United States based on wholesale acquisition cost pricing on January 2016 reported lifetime patient costs of palbociclib plus letrozole combination therapy to be $\$ 372,761$ while providing 2.13 quality-adjusted life years. ${ }^{52}$ The lifetime patient costs of letrozole monotherapy were $\$ 128,435$ while providing an average of 1.82 quality-adjusted life years. ${ }^{52}$ Thus, palbociclib plus letrozole therapy at prices in January 2016 cost \$768,498 more per quality-adjusted life year than letrozole monotherapy. ${ }^{52}$

Two years later, with two more CDK4/6 inhibitors on the market, costs have only increased. ${ }^{49-51,53}$ To offset these costs, all three pharmaceutical companies have established programs to reduce patient costs from wholesale acquisition costs. Palbociclib and ribociclib are both attainable to some uninsured or low-income patients at costs ranging from $\$ 0$ to $\$ 10$ per month. ${ }^{53-55}$ Commercially insured patients can qualify for savings of up to $\$ 25,000$ per year with palbociclib or abemaciclib. Ribociclib offers a copack of ribociclib and letrozole tablets at the same cost as ribociclib alone, with some commercially insured patients qualifying for savings of up to $\$ 15,000$ per product per year. ${ }^{54}$ Palbociclib is supplied at three doses of 125,100 , and $75 \mathrm{mg}$, while abemaciclib is supplied at four doses of $200,150,100$, and $50 \mathrm{mg}$, with a 1-month supply priced the same regardless of dose level. ${ }^{49,51}$ When dose reductions are needed for either of these, patients may need to discard part of their expensive monthly supply and order the next lower dose bottle. ${ }^{12,41}$ Ribociclib is formulated in $200 \mathrm{mg}$ tablets with dose reduction requiring taking one fewer tablet per day. ${ }^{11}$ Thus, 400 and $200 \mathrm{mg}$ dose packs are priced proportionally lower than the starting $600 \mathrm{mg}$ dose, which could result in reduced costs for some patients. ${ }^{50}$ Currently, the only support for patients on Medicare and Medicaid insurance is conditional support for palbociclib if no charitable support is available. ${ }^{54-56}$ Taking eligibility for price reductions into account, financial costs should be considered alongside efficacy and safety when prescribing CDK4/6 inhibitor plus AI therapies.

\section{Predictive biomarkers}

The barriers in this review have likely limited the use of CDK4/6 inhibitors in first- and second-line treatment of $\mathrm{HR}+$, HER2-ABC more than might be anticipated given the favorable Phase III data available. In addition to these barriers, the current lack of readily available predictive biomarkers to identify marked sensitivity or resistance to these compounds is an impediment to their use. Preclinical studies in tumor cell models have suggested that breast tumors may be resistant to CDK4/6 inhibitor therapy if they contain certain mutations in the cell-cycle pathway, such as changes in cyclin D1 or cyclin E1 expression or loss of retinoblastoma protein $(\mathrm{Rb}) .{ }^{57-60}$ Genetic loss of $\mathrm{Rb}$ occurs in only $2 \%$ of breast cancer cases, and Phase III trials of CDK4/6 inhibitors have not reported results in this patient subgroup. ${ }^{61}$ Clinical trial analyses have investigated the effect of baseline mutations on the efficacy of CDK4/6 inhibitors; however, these results may be limited by the small number of enrolled patients in some subgroups and duration of follow-up. ${ }^{62-64}$ Additionally, no diagnostic tools are available to clinicians to assess these biomarkers in the real-world setting, restricting the ability of clinicians to apply results to clinical practice. Nevertheless, CDK4/6 inhibitor plus AI therapy has demonstrated improved PFS over AI monotherapy in many patient subgroups split by baseline biomarker phenotypes enrolled in the PALOMA-1, PALOMA-2, and MONALEESA-2 trials.

From the PALOMA-1 and PALOMA-2 trials, PFS benefit of palbociclib plus letrozole was observed in patients irrespective of $C C N D 1, C D K 4, C D K 6, R B 1, C D K N 2 A$, $C C N E 1, C C N E 2, C D K 2, C C N D 3, E S R 1$, or $A R$ gene expression or Ki67 or ER protein expression. ${ }^{62,65,66}$ This benefit was also observed in patients with high gene expression of $P D C D 1$, high protein expression of $\mathrm{Rb}, \mathrm{p} 16$, or cyclin $\mathrm{D} 1$, or irrespective of $\mathrm{Rb}$ localization or $\mathrm{p} 16$ nuclear expression levels. ${ }^{62,65,66}$ Small sample sizes restrict conclusions made about efficacy in tumors with low p16, Rb, or cyclin D1 protein expression. ${ }^{45,62}$

From the MONALEESA-2 trial, PFS benefit of ribociclib plus letrozole combination therapy was observed in patients irrespective of $\mathrm{p} 16$ protein expression or PIK3CA mutation status and in patients with high gene expression of $E S R$ l, low gene expression of $C D K N 2 A$ or $C C N D 1$, and high protein expression of $\mathrm{Rb}$ or $\mathrm{Ki} 67 .{ }^{64}$ However, small sample sizes and 
limited follow-up restricted conclusions made from analyses of other expression levels of these proteins and genes. ${ }^{64}$

Even in patients with baseline genetic changes that promote resistance to CDK4/6 inhibitor plus AI therapy, patients may achieve a PFS benefit with CDK4/6 inhibitor plus AI therapy over AI monotherapy. For example, baseline mutations in TP53, which encodes the tumor suppressor protein p53, have been associated with resistance to abemaciclib and early progression on palbociclib and ribociclib. ${ }^{63,67,68}$ However, in the MONALEESA-2 trial in patients with mutant TP53, a PFS benefit was observed with ribociclib plus letrozole combination therapy over letrozole monotherapy. ${ }^{63}$

Evidence from ongoing trials suggests that CDK4/6 inhibitor-based combination therapy can provide benefit to patients who have resistance to prior therapies. The TREnd trial reported that patients whose disease had progressed after $>6$ months of ET in the advanced setting demonstrated a significant improvement in median PFS with palbociclib plus the same ET vs palbociclib alone (11.5 months vs 6.5 months, $P=0.02) .{ }^{69,70}$ Furthermore, the Phase I/II TRINITI-1 trial reported that patients whose disease had progressed after CDK4/6 inhibitor-based therapy experienced a benefit of ribociclib in combination with everolimus and exemestane. ${ }^{71}$ A similar Phase II trial, BioPER, is recruiting patients with metastatic breast cancer who had experienced clinical benefit with palbociclib plus ET and had progressed on the treatment 3-8 weeks before study entry. ${ }^{72}$ While no results have yet been reported, the trial will assess biomarkers of resistance and clinical benefit of palbociclib plus ET in the post-palbociclib setting. These trials begin to address questions of sequencing after resistance to CDK4/6 inhibitor therapy occurs.

\section{Conclusion}

Despite the gains in efficacy, some physicians are still reluctant to use $\mathrm{CDK} 4 / 6$ inhibitors in combination with an $\mathrm{AI}$ as first-line treatment in postmenopausal women with HR+, HER2- ABC because of concerns about efficacy, safety, and cost. However, our review of the data indicates that CDK4/6 inhibitors improve PFS in all eligible patients, with comparable response rates to first-line chemotherapy. CDK4/6 inhibitors in combination with AIs result in a greater number of AEs compared to AI monotherapy; however, patient-reported QoL is similar between treatment groups, suggesting that AEs are manageable with recommended monitoring. Patient costs remain a barrier with three CDK4/6 inhibitors on the market, but patient support programs may greatly reduce these costs for some patients, and assessment of this barrier should be on a case-by-case basis alongside desired efficacy and safety concerns.
While no changes in mRNA or protein expression that predict resistance to CDK4/6 inhibitors in the clinical setting have been found, further research is needed to identify mechanisms of resistance to CDK4/6 inhibitor plus AI therapy. Ongoing trials may provide valuable insights into continued use of CDK4/6 inhibitors after resistance to first-line combination therapy develops. As the breadth of studies of CDK4/6 inhibitors continues to expand, physicians should ensure that they consider current clinical trial data alongside individual patient needs in their treatment decisions.

\section{Acknowledgments}

Editorial assistance was provided under the direction of the authors by Ted Stanek, PhD, and David Boffa, ELS, MedThink SciCom, with support from Novartis Pharmaceuticals Corporation. Ribociclib was discovered by Novartis Institutes for BioMedical Research in collaboration with Astex Pharmaceuticals.

\section{Author contributions}

Dr Mahtani and Dr Vogel conceived of and designed the review, revised it critically for important intellectual content, and approved the final version to be published. Both authors contributed to data analysis, drafting and revising the article, gave final approval of the version to be published, and agree to be accountable for all aspects of the work.

\section{Disclosure}

Dr Mahtani served as a consultant/advisor to Pfizer, Novartis, Celgene, Genentech, Lilly, Eisai, and Amgen and received research support from Genentech. Dr Vogel served as a consultant/advisor to Adgero Biopharmaceuticals and GTx, Inc. The authors report no other conflicts of interest in this work.

\section{References}

1. National Cancer Institute Surveillance, Epidemiology, and End Results Program [webpage on the Internet]. Cancer Stat Facts: Female Breast Cancer; 2018. Available from: https://seer.cancer.gov/statfacts/html/ breast.html. Accessed July 12, 2018.

2. Blows FM, Driver KE, Schmidt MK, et al. Subtyping of breast cancer by immunohistochemistry to investigate a relationship between subtype and short and long term survival: a collaborative analysis of data for 10,159 cases from 12 studies. PLoS Med. 2010;7(5):e1000279.

3. Rugo HS, Rumble RB, Macrae E, et al. Endocrine therapy for hormone receptor-positive metastatic breast cancer: American Society of Clinical Oncology Guideline. J Clin Oncol. 2016;34(25):3069-3103.

4. Partridge AH, Rumble RB, Carey LA, et al. Chemotherapy and targeted therapy for women with human epidermal growth factor receptor 2-negative (or unknown) advanced breast cancer: American Society of Clinical Oncology Clinical Practice Guideline. J Clin Oncol. 2014;32(29):3307-3329. 
5. Reinert T, Debiasi M, Bines J, Barrios CH. Trends in progression-free survival (PFS) and time to progression (TTP) over time within first-line aromatase inhibitors trials in hormone receptor-positive advanced breast cancer. Breast Cancer Res Treat. 2018;168(2):457-465.

6. Finn RS, Crown JP, Lang I, et al. The cyclin-dependent kinase 4/6 inhibitor palbociclib in combination with letrozole versus letrozole alone as first-line treatment of oestrogen receptor-positive, HER2-negative, advanced breast cancer (PALOMA-1/TRIO-18): a randomised phase 2 study. Lancet Oncol. 2015;16(1):25-35.

7. Finn RS, Martin M, Rugo HS, et al. Palbociclib and letrozole in advanced breast cancer. $N$ Engl J Med. 2016;375(20):1925-1936.

8. Goetz MP, Toi M, Campone M, et al. MONARCH 3: Abemaciclib as initial therapy for advanced breast cancer. J Clin Oncol. 2017;35(32):3638-3646.

9. Hortobagyi GN, Stemmer SM, Burris HA, et al. Updated results from MONALEESA-2, a phase III trial of first-line ribociclib plus letrozole versus placebo plus letrozole in hormone receptor-positive, HER2negative advanced breast cancer. Ann Oncol. 2018;29(7):1541-1547.

10. Tripathy D, Im SA, Colleoni M, et al. Ribociclib plus endocrine therapy for premenopausal women with hormone-receptor-positive, advanced breast cancer (MONALEESA-7): a randomised phase 3 trial. Lancet Oncol. 2018;19(7):904-915.

11. Kisqali [package insert]. East Hanover, NJ: Novartis Pharmaceuticals Corporation; 2018.

12. Ibrance [package insert]. New York, NY: Pfizer; 2018.

13. Beaver JA, Amiri-Kordestani L, Charlab R, et al. FDA Approval: palbociclib for the treatment of postmenopausal patients with estrogen receptor-positive, HER2-negative metastatic breast cancer. Clin Cancer Res. 2015;21(21):4760-4766.

14. Burstein HJ, Mayer EL, DeMichele A, et al. Treatment patterns for young women with HR+/HER2- metastatic breast cancer in the United States in the era of CDK 4/6 inhibitors. San Antonio Breast Cancer Symposium; December 5-9, 2017; San Antonio, TX.

15. Zanotti G, Hunger M, Perkins JJ, Horblyuk R, Martin M. Treatment patterns and real world clinical outcomes in ER+/HER2- post-menopausal metastatic breast cancer patients in the United States. BMC Cancer. 2017;17(1):393.

16. Hortobagyi GN, Stemmer SM, Burris HA, et al. Ribociclib as firstline therapy for HR-positive, advanced breast cancer. $N$ Engl $J$ Med. 2016;375(18):1738-1748

17. Rugo HS, Finn RS, Dieras V, et al. Palbociclib plus letrozole as firstline therapy in estrogen receptor-positive/human epidermal growth factor receptor 2-negative advanced breast cancer: efficacy and safety updates with longer follow-up across patient subgroups. San Antonio Breast Cancer Symposium; December 5-7, 2017; San Antonio, TX.

18. Rugo HS, Turner NC, Finn RS, et al. Palbociclib plus endocrine therapy in older women with HR+/HER2- advanced breast cancer: a pooled analysis of randomised PALOMA clinical studies. Eur $J$ Cancer. 2018;101:123-133.

19. Sonke GS, Hart LL, Campone M, et al. Ribociclib with letrozole vs letrozole alone in elderly patients with hormone receptor-positive, HER2-negative breast cancer in the randomized MONALEESA-2 trial. Breast Cancer Res Treat. 2018;167(3):659-669.

20. Singh H, Howie L, Bloomquist E, et al. U.S. Food and Drug Administration pooled analysis of outcomes of older women with hormone-receptor positive metastatic breast cancer treated with a CDK4/6 inhibitor as initial endocrine based therapy. San Antonio Breast Cancer Symposium; December 5-9, 2017; San Antonio, TX.

21. Lee SJ, Park S, Ahn HK, et al. Implications of bone-only metastases in breast cancer: favorable preference with excellent outcomes of hormone receptor positive breast cancer. Cancer Res Treat. 2011;43(2): 89-95.

22. Schröder J, Fietz T, Köhler A, et al. Treatment and pattern of bone metastases in 1094 patients with advanced breast cancer - results from the prospective German Tumour Registry Breast Cancer cohort study. Eur J Cancer. 2017;79:139-148.
23. Sledge GW, Toi M, Neven P, et al. MONARCH 2: abemaciclib in combination with fulvestrant in women with HR+/HER2- advanced breast cancer who had progressed while receiving endocrine therapy. J Clin Oncol. 2017;35(25):2875-2884.

24. Turner NC, Finn RS, Martin M, et al. Clinical considerations of the role of palbociclib in the management of advanced breast cancer patients with and without visceral metastases. Ann Oncol. 2018;29(3):669-680.

25. Goetz MP, O'Shaughnessy J, Sledge GW, et al. The benefit of abemaciclib in prognostic subgroups: an exploratory analysis of combined data from the MONARCH 2 and 3 studies. San Antonio Breast Cancer Symposium; December 5-9, 2017; San Antonio, TX.

26. Burris HA, Chan A, Campone M, et al. First-line ribociclib + letrozole in patients with HR+, HER2- advanced breast cancer presenting with visceral metastases or bone-only disease: a subgroup analysis of the MONALEESA-2 trial. San Antonio Breast Cancer Symposium; December 6-10, 2016; San Antonio, TX.

27. Finn RS, Dieras V, Rugo HS, et al. Palbociclib plus letrozole as first-line therapy in estrogen receptor-positive/human epidermal growth factor receptor 2-negative advanced breast cancer: efficacy and safety across patient subgroups. American Society of Clinical Oncology Annual Meeting; June 2-6, 2017; Chicago, IL.

28. Janni W, Alba E, Bachelot T, et al. First-line ribociclib plus letrozole in postmenopausal women with HR+, HER2- advanced breast cancer: tumor response and pain reduction in the phase 3 MONALEESA-2 trial. Breast Cancer Res Treat. 2018;169(3):469-479.

29. Tripathy D, Sohn J, Im S-A, et al. First-line ribociclib or placebo combined with goserelin and tamoxifen or a non-steroidal aromatase inhibitor in premenopausal women with hormone receptor-positive, HER2-negative advanced breast cancer: results from the randomized Phase III MONALEESA-7 trial. San Antonio Breast Cancer Symposium; December 5-9, 2017; San Antonio, TX.

30. Gradishar WJ, Krasnojon D, Cheporov S, et al. Significantly longer progression-free survival with nab-paclitaxel compared with docetaxel as first-line therapy for metastatic breast cancer. J Clin Oncol. 2009;27(22):3611-3619.

31. Katsumata N, Watanabe T, Minami H, et al. Phase III trial of doxorubicin plus cyclophosphamide (AC), docetaxel, and alternating $\mathrm{AC}$ and docetaxel as front-line chemotherapy for metastatic breast cancer: Japan Clinical Oncology Group trial (JCOG9802). Ann Oncol. 2009;20(7):1210-1215.

32. Nabholtz JM, Gelmon K, Bontenbal M, et al. Multicenter, randomized comparative study of two doses of paclitaxel in patients with metastatic breast cancer. J Clin Oncol. 1996;14(6):1858-1867.

33. O'Shaughnessy J, Miles D, Vukelja S, et al. Superior survival with capecitabine plus docetaxel combination therapy in anthracyclinepretreated patients with advanced breast cancer: phase III trial results. J Clin Oncol. 2002;20(12):2812-2823.

34. Rocca A, Schirone A, Maltoni R, et al. Progress with palbociclib in breast cancer: latest evidence and clinical considerations. Ther $A d v$ Med Oncol. 2017;9(2):83-105.

35. Robertson JFR, Di Leo A, Fazal M, Lichfield J, Ellis MJ. Fulvestrant for hormone receptor-positive advanced breast cancer in patients with visceral versus non-visceral metastases: findings from FALCON, FIRST, AND CONFIRM. San Antonio Breast Cancer Symposium; December 5-9, 2017; San Antonio, TX.

36. Sammons SL, Topping DL, Blackwell KL. HR+, HER2- advanced breast cancer and CDK4/6 inhibitors: mode of action, clinical activity, and safety profiles. Curr Cancer Drug Targets. 2017;17(7):637-649.

37. Smith TJ, Bohlke K, Lyman GH, et al. Recommendations for the use of WBC growth factors: American Society of Clinical Oncology clinical practice guideline update. J Clin Oncol. 2015;33(28):3199-3212.

38. Dieras V, Harbeck N, Joy AA, et al. PALOMA-2: Neutropenia patterns in patients with estrogen receptor-positive/human epidermal growth factor receptor 2-negative first-line advanced breast cancer receiving palbociclib plus letrozole. European Society for Medical Oncology Congress; September 8-12, 2017; Madrid, Spain. 
39. Janni W, Burris HA, Blackwell KL, et al. First-line ribociclib + letrozole for postmenopausal women with HR+, HER2-ABC: MONALEESA-2 safety results. American Society of Clinical Oncology Annual Meeting; June 2-6, 2017; Chicago, IL.

40. Finn RS, Crown JP, Ettl J, et al. Efficacy and safety of palbociclib in combination with letrozole as first-line treatment of ER-positive, HER2negative, advanced breast cancer: expanded analyses of subgroups from the randomized pivotal trial PALOMA-1/TRIO-18. Breast Cancer Res. 2016;18(1):67.

41. Verzenio [package insert]. Indianapolis, IN: Eli Lilly and Company; 2018.

42. US. Department of Health and Human Services. Common Terminology Criteria for Adverse Events (CTCAE) Version 5.0. Available from: https://ctep.cancer.gov/protocoldevelopment/electronic_applications/ docs/CTCAE_v5_Quick_Reference_5x7.pdf. Accessed March 6, 2018.

43. Vandenberk B, Vandael E, Robyns T, et al. Which QT correction formulae to use for QT monitoring? J Am Heart Assoc. 2016;5(6): e003264.

44. Drew BJ, Ackerman MJ, Funk M, et al. Prevention of torsade de pointes in hospital settings: a scientific statement from the American Heart Association and the American College of Cardiology Foundation. $J$ Am Coll Cardiol. 2010;55(9):934-947.

45. ClinicalTrials.gov. A study of palbociclib (PD-0332991)+ letrozole vs. letrozole for 1st line treatment of postmenopausal women with ER+/ HER2- advanced breast cancer (PALOMA-2). Available from: https:// www.clinicaltrials.gov/ct2/show/results/NCT01740427. NLM identifier: NCT01740427 Accessed January 18, 2017

46. Rugo HS, Diéras V, Gelmon KA, et al. Impact of palbociclib plus letrozole on patient-reported health-related quality of life: results from the PALOMA-2 trial. Ann Oncol. 2018;29(4):888-894.

47. Bell T, Crown JP, Lang I, et al. Impact of palbociclib plus letrozole on pain severity and pain interference with daily activities in patients with estrogen receptor-positive/human epidermal growth factor receptor 2-negative advanced breast cancer as first-line treatment. Curr Med Res Opin. 2016;32(5):959-965.

48. Verma S, O'Shaughnessy J, Burris HA, et al. Health-related quality of life of postmenopausal women with hormone receptor-positive, human epidermal growth factor receptor 2-negative advanced breast cancer treated with ribociclib+letrozole: results from MONALEESA-2. Breast Cancer Res Treat. 2018;170(3):535-545.

49. GoodRx.com [database on the Internet]. Palbociclib (Ibrance). Available from: https://www.goodrx.com/palbociclib. Accessed August 23, 2018.

50. GoodRx.com [database on the Internet]. Ribociclib (Kisqali). Available from: https://www.goodrx.com/ribociclib. Accessed August 23, 2018.

51. GoodRx.com [database on the Internet]. Abemaciclib (Verzenio). Available from: https://www.goodrx.com/abemaciclib. Accessed August 23 , 2018.

52. Mamiya H, Tahara RK, Tolaney SM, Choudhry NK, Najafzadeh M. Cost-effectiveness of palbociclib in hormone receptor-positive advanced breast cancer. Ann Oncol. 2017;28(8):1825-1831.

53. Mustacchi G, Generali D. Cost-effectiveness and sustainability of breast cancer screening and new anti-cancer drugs. J Med Econ. 2017;20(4):405-408.

54. Novartis Pharmaceuticals Corporation. Financial Resources. Available from: https://www.us.kisqali.com/metastatic-breast-cancer/patientsupport/financial-resources/. Accessed August 23, 2018.

55. Pfizer Corporation [database on the Internet]. Financial Assistance. Available from: https://www.ibrance.com/financial-assistance. Accessed August 23, 2018.

56. Eli Lilly and Company. Savings Support [database on the Internet]. Available from: https://www.verzenio.com/savings-support. Accessed August 23, 2018.

57. Herrera-Abreu MT, Palafox M, Asghar U, et al. Early adaptation and acquired resistance to $\mathrm{CDK} 4 / 6$ inhibition in estrogen receptor-positive breast cancer. Cancer Res. 2016;76(8):2301-2313.

58. Knudsen ES, Witkiewicz AK. The strange case of CDK4/6 inhibitors: mechanisms, resistance, and combination strategies. Trends Cancer. 2017;3(1):39-55.
59. Lenihan C, Bouchekioua-Bouzaghou K, Abdulghani R, Chupin J, Shia A, Schmid P. CDK4/6 inhibitor resistant ER-positive cells remain dependent on estrogen signalling and retain sensitivity to endocrine therapy. San Antonio Breast Cancer Symposium; December 6-10, 2016; San Antonio, TX.

60. Xiao Y, Ong CC, Daemen A, Friedman L, O’Brien T. Identification of preclinical mechanisms driving acquired resistance to selective ERa degraders (SERDs), CDK4/6 inhibitors, or to combinations of both agents. San Antonio Breast Cancer Symposium; December 6-10, 2016; San Antonio, TX.

61. Cancer Genome Atlas Network. Comprehensive molecular portraits of human breast tumours. Nature. 2012;490(7418):61-70.

62. Finn R, Jiang Y, Rugo H, et al. Biomarker analyses from the phase 3 PALOMA-2 trial of palbociclib (P) with letrozole (L) compared with placebo (PLB) plus L in postmenopausal women with ER+/HER2advanced breast cancer (ABC). European Society for Medical Oncology Congress; October 7-11, 2016; Copenhagen, Denmark.

63. Hortobagyi GN, Stemmer SM, Campone M, et al. First-line ribociclib + letrozole in hormone receptor-positive, HER2-negative advanced breast cancer: efficacy by baseline circulating tumor DNA alterations in MONALEESA-2. San Antonio Breast Cancer Symposium; December 5-9, 2017; San Antonio, TX.

64. Andre F, Stemmer SM, Campone M, et al. Ribociclib + letrozole for first-line treatment of HR+, HER2- advanced breast cancer: efficacy by baseline tumor markers. American Association for Cancer Research Annual Meeting; April 1-5, 2017; Washington, DC.

65. Finn RS, Liu Y, Martin M, et al. Comprehensive gene expression biomarker analysis of cyclin-dependent kinases 4/6 and endocrine pathways from the PALOMA-2 study. San Antonio Breast Cancer Symposium; December 5-9, 2017; San Antonio, TX.

66. Demichele A, Clark AS, Tan KS, et al. CDK 4/6 inhibitor palbociclib (PD0332991) in Rb+ advanced breast cancer: phase II activity, safety, and predictive biomarker assessment. Clin Cancer Res. 2015;21(5): 995-1001

67. Cruz MRS, Behdad A, Strickland K, et al. Patterns of genomic alterations in ER-positive advanced breast cancer patients treated with CDK4/6 inhibitors. San Antonio Breast Cancer Symposium; December 5-9, 2017; San Antonio, TX.

68. Patnaik A, Rosen LS, Tolaney SM, et al. Efficacy and safety of abemaciclib, an inhibitor of CDK4 and CDK6, for patients with breast cancer, non-small cell lung cancer, and other solid tumors. Cancer Discov. 2016;6(7):740-753.

69. Malorni L, Curigliano G, Minisini A, et al. A phase II trial of the CDK4/6 inhibitor Palbociclib $(\mathrm{P})$ as single agent or in combination with the same endocrine therapy (ET) received prior to disease progression, in patients (pts) with hormone receptor positive (HR+) HER2 negative (HER2-) metastatic breast cancer (mBC) (TREnd trial). American Society of Clinical Oncology Annual Meeting; June 2-6, 2017; Chicago, IL.

70. Malorni L, Curigliano G, Minisini AM, et al. Palbociclib as single agent or in combination with the endocrine therapy received before disease progression for estrogen receptor-positive, HER2-negative metastatic breast cancer: TREnd trial. Ann Oncol. 2018;29(8):1748-1754.

71. Moulder S, Karuturi M, Yardley DA, et al. Ribociclib in combination with everolimus and exemestane in men and postmenopausal women with $\mathrm{HR}+/ \mathrm{HER} 2-$ advanced breast cancer after progression on a CDK4/6 inhibitor: efficacy and safety results from Phase 2 of the TRINITI-1 study. American Association for Cancer Research; April 14-18, 2018; Chicago, IL.

72. ClinicalTrials.gov. Molecular Mechanisms of Resistance and Sensitivity to Palbociclib Re-challenge in ER+ mBC (BioPER). Available from: https:/www.clinicaltrials.gov/ct2/show/NCT03184090. NLM identifier: NCT03184090 Accessed August 24, 2018.

73. Blackwell KL, Paluch-Shimon S, Campone M, et al. Subsequent treatment for postmenopausal women with hormone receptor-positive, HER2-negative advanced breast cancer who received ribociclib + letrozole vs placebo + letrozole in the Phase III MONALEESA-2 study. San Antonio Breast Cancer Symposium; December 5-9, 2017; San Antonio, TX. 


\section{Publish your work in this journal}

Cancer Management and Research is an international, peer-reviewed open access journal focusing on cancer research and the optimal use of preventative and integrated treatment interventions to achieve improved outcomes, enhanced survival and quality of life for the cancer patient. The manuscript management system is completely online and includes a very quick and fair peer-review system, which is all easy to use. Visit http://www.dovepress.com/testimonials.php to read real quotes from published authors.

Submit your manuscript here: https://www.dovepress.com/cancer-management-and-research-journal 